\title{
Design on Urban Water Supply Pipe Network Accident Reasoning System based on FCR
}

\author{
Yongchang Ren ${ }^{1, a}$, Jiao Zhang ${ }^{1, b}$, Ying Zhao ${ }^{2, c}$, Chunqiang $\mathrm{Li}^{3, \mathrm{~d}}$ \\ ${ }^{1}$ College of Information Science and Technology, Bohai University, Jinzhou, 121013, China \\ ${ }^{2}$ Computer Center, Liaoning University of Technology, Jinzhou, 121013, China \\ ${ }^{3}$ School of Information Management, Beijing Information Science and Technology University, \\ Beijing, 100101, China \\ a1213552916@qq.com, b593599610@qq.com, 'Igjsjzy8@163.com, '642510571@qq.com
}

Keywords: FCR; urban water supply pipe network; accident; reasoning system; fuzzy-based reasoning; case-based reasoning; rule-based reasoning; function structure

\begin{abstract}
In order to do a good job in water supply network accident emergency need to build a perfect emergency decision system, improve the disposal of the response capacity of pipe network accident, when the pipeline accidents timely and effectively organize manpower and material resources. According to the characteristics of the urban water supply pipeline accident is difficult to detect, assistant decision-making system design based on the FCR reasoning. Using the technology, based on the fuzzy reasoning based on case reasoning skills and rule-based reasoning technology, three kinds of technologies such as hybrid reasoning mechanism, play the advantages of three kinds of technology, to overcome the shortcomings of traditional reasoning technology, to detect whether the water supply network accident as the ultimate goal, overcome the shortcomings of traditional reasoning technology, at the same time improve the efficiency and effect of reasoning system.
\end{abstract}

\section{Introduction}

Water supply pipe network safe operation is an important link in ensuring the safety of city water supply, involved in homes and to create civilized city morale project, is also the important of the construction of a harmonious society and safeguard social stability. Thus making perfect the emergency response system, improve the response capacity of processing pipeline accidents, accidents occurred in the network organization resources, manpower and material resources in a timely and effective manner when doing a water supply pipeline emergency repair work is more urgent and important. For emergency response speed and decision-making capacity is the important symbol of urban modernization level, is also the main indicators of government emergency management effectiveness.

Emergency decision making in the face of uncertain dynamic environment, including emergency event occurrence, development and evolution of uncertainty, emergency treatment effect of uncertainty, emergency measures in time, the uncertainty on the resource consumption, etc. Therefore, it is necessary in the different stages of emergency situation on the basis of scientific evaluation, the uncertainty decision-making model and method. Urban water supply pipeline accident disposal level of the ability of fast response and decision, will directly decide the size of its negative effects. Scientific emergency decision-making method can make the different institutions to make timely and effective response, and in the event of circumstances, each decision-making can according to your own decision goal and effective decision-making procedures for rapid transformation, finally realizes the emergency disposal of high efficiency and high speed. This article is based on the fusion of fuzzy, case and rules reasoning study, for the urban water supply network disposal decision provides new theory and method. 


\section{Fuzzy-Based Reasoning}

Fuzzy -based Reasoning (FBR) is used to simulate human thinking process daily, also known as the approximate Reasoning and Fuzzy knowledge and Fuzzy information, Fuzzy logic Reasoning method, belonging to the uncertainty Reasoning. In some systems, not all the characteristics of the attribute values are certain, so using a simple accurate value will lose a lot of information. In this case, the FBR technology never need accurate precondition of reasoning that may not accurate conclusion. FBR technology not only has been widely used in many traditional areas, and the successful application of the emerging field of intelligent control, including fuzzy control, fuzzy expert system and fuzzy pattern recognition, etc.

FBR is the fuzzy-based reasoning, is the mapping process from input to output, reasoning process is shown in Fig. 1. Specific steps are as follows: the input to the variable fuzzy processing, the input of precise variable fuzzy variables are a, by using membership function of fuzzy variables; Activate the rules of the fuzzy rule base, the use of appropriate fuzzy implication operator and fuzzy synthesis operator calculation results, the final output comprehensive fuzzy set; In the second step to deal with the blur comprehensive fuzzy set, so as to get a precise output.

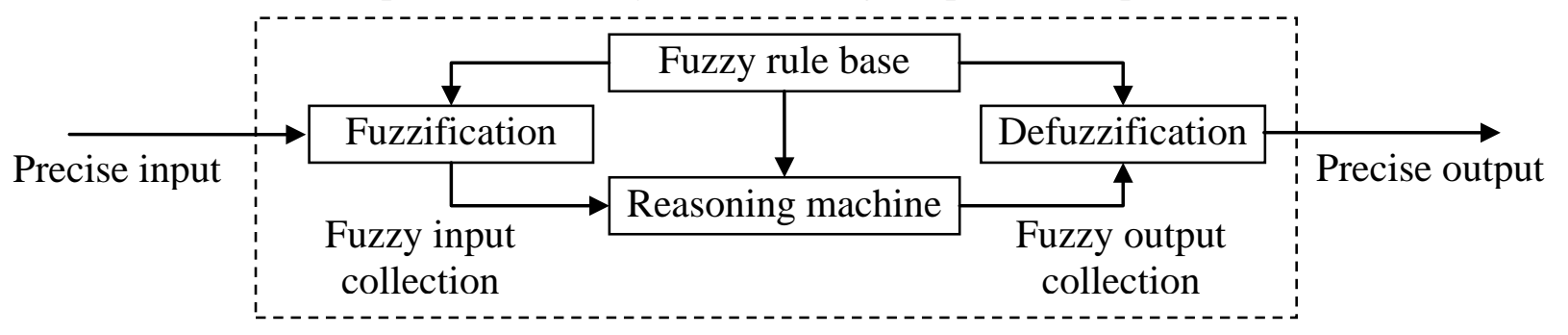

Fig. 1. Fuzzy reasoning process

\section{Case-Based Reasoning}

Case -based Reasoning (CBR), by looking for similar Case history, the use of experience or the results of specific knowledge that is specific Case to solve the new problems. CBR research methods derived from human cognitive psychological activity, alleviate the conventional knowledge acquisition bottleneck problems in knowledge system, combining quantitative analysis with qualitative analysis, has the characteristics of the dynamic knowledge base and incremental learning. CBR is usually divided into interpreted case-based reasoning of case-based reasoning and problem solving. Interpreted the case-based reasoning, as a reference to previous cases to categorize the situation, describe and explain; Problem solving model of case-based reasoning. Using the previous cases put forward constructive solutions to the current problems.

Typical case reasoning problem solving process, the basic steps can be summarized as four main processes: case retrieval, case reuse and case modification and case. Work process is shown in Fig. 2. In case-based reasoning, the problems to be resolved or condition usually referred to as the target case, called the case history source case, the source of the case collection called putted forward. In addition, people often according to the case of new cases and in case the similarity and difference between, to determine how to adjust for the solution to the existing case, to adapt to the new environment and problems.

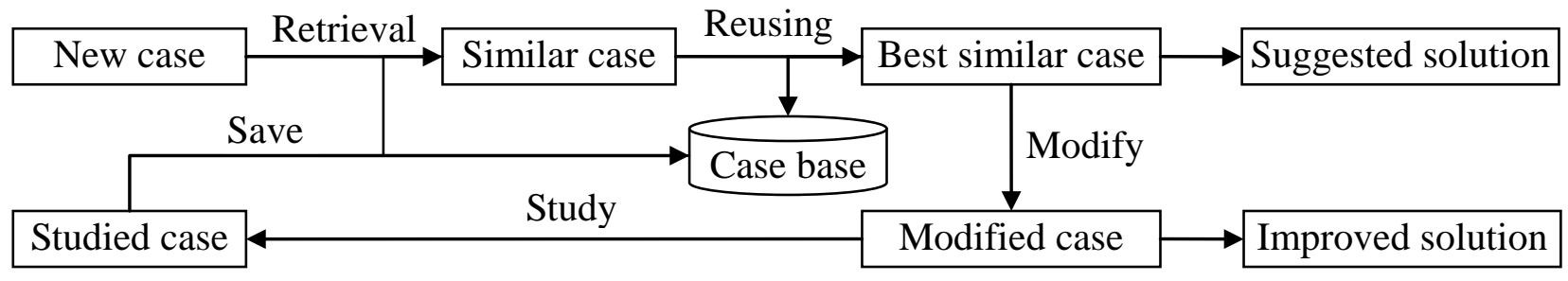

Fig. 2. Case reasoning process 


\section{Rule-Based Reasoning}

Rule -based Reasoning (RBR), refers to the relevant experts in the field of knowledge formalization description, form the system of rules. These rules said some of the problems in this field and the corresponding answer these questions, and you can use rules to imitate the experts in solving the related reasoning. Rule is based on the domain constraint expression, with a number of conditions, at the same time defines these conditions are satisfied circumstances should take action, as a result, the rule is a logic process, can be described with a logical volume. At the same time because of the need to adapt to rules system approach, rules engine technical requirements is production rules. Production rules based on formal language accurate description of abstract structure build rules.

According to the nature and characteristics of the rule engine technology, there are two kinds of suitable rules system reasoning: forward reasoning, THEN from the IF part of the part of the reasoning process, from the fact or condition to the target or action, is the imitation of human forward reasoning, execution process is driven by data. Reverse reasoning, from the part THEN to the IF part of the reasoning process, from the target or action to the fact or condition, is the imitation of human backward inference; Forward inference and backward inference, the main work is to search, both of the rules of search and query of facts, can understand as a variant form of search. Involved in the process of search compared two kinds of reasoning mechanism by pattern matching, so the pattern matching algorithm is basis of reasoning rules engine technology.

\section{FCR Fusion Reasoning}

In the field of artificial intelligence, based on Fuzzy Reasoning technique (FBR, Fuzzy - -based Reasoning), case-based Reasoning (CBR, Case -Based Reasoning) and rule-based Reasoning (RBR, Rule - -based Reasoning) is a typical method of Reasoning to solve problems. Existing in the accident of inference system often use only one or two kinds of reasoning technology, there is no denying that these reasoning technology has more advantages, but also difficult in knowledge acquisition, real-time performance and the lack of methods of solving the faults is determined, considering these deficiencies, this paper tries to study the mechanism of three kinds of technology integration, the FBR, CBR and RBR, and these three fusion reasoning methods, establish a hybrid reasoning mechanism, hereinafter referred to as fusion technology for FCR reasoning.

Reasoning machine (Inference Engine) is implemented in expert system based on knowledge reasoning components, is based on the knowledge reasoning in the computer implementation, mainly including reasoning and control two aspects, is an important part of knowledge in the system. Reasoning machine is mainly composed of actuators, the scheduler and consistency coordinator, etc. Main machine is the key to realize the FCR reasoning, will implement the contingency plan design of water supply pipe reasoning process of the overall scheduling function, its task is to control the sequence of reasoning machine reasoning as well as between the invocation of the process, with the aid of fuzzy knowledge, case and rule reasoning part together complete the call between different knowledge base and internal reasoning to solve. Reasoning structure as is shown in Fig. 3.

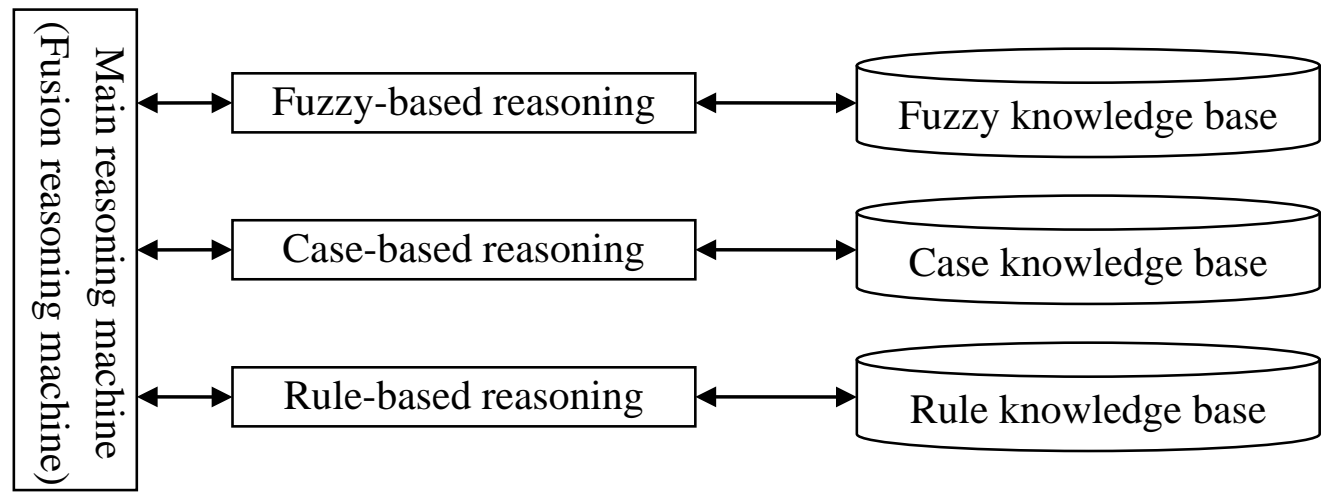

Fig. 3. FCR reasoning structure 
For FCR reasoning algorithm is shown in Fig.3 can be described as: calling sequence can be fuzzy inference machine, case-based reasoning and rule reasoning machine; Can also be a rule reasoning machine to case-based reasoning machine, fuzzy inference machine; Can also according to the pipeline accident emergency disposal of the particular case just call one or two of three kinds of reasoning machine. Each function call between reasoning machine, the first to advocate the principle request, by the main machine call the corresponding function reasoning machine, and the reasoning results back to the main machine for subsequent reasoning.

Normal operation of water supply network, can't guarantee the stability of all the attributes of keep on a numeric value, will be in a range of floating up and down, the float may be normal, also is likely to be abnormal, the abnormal may herald an accident. The difficulty of fuzzy similarity calculation is FCR reasoning. When the attribute value features of water supply network, a fuzzy number, cannot use the traditional similarity calculation method, but need to define a new kind of, in view of the fuzzy numerical similarity calculation method.

\section{System Function Structure}

To build an efficient and perfect water supply network inference system accident, you must have a perfect overall design, and it not only can satisfy the requirements of the network complexity etc. and can rapidly and accurately for reasoning. The accident system mainly includes the following several modules, respectively, personnel management module, the fusion of CBR and RBR reasoning module, the fusion of FBR and RBR reasoning module, management module, the rule base case management module, and system help module. The System Function Structure as shown in Fig.4.

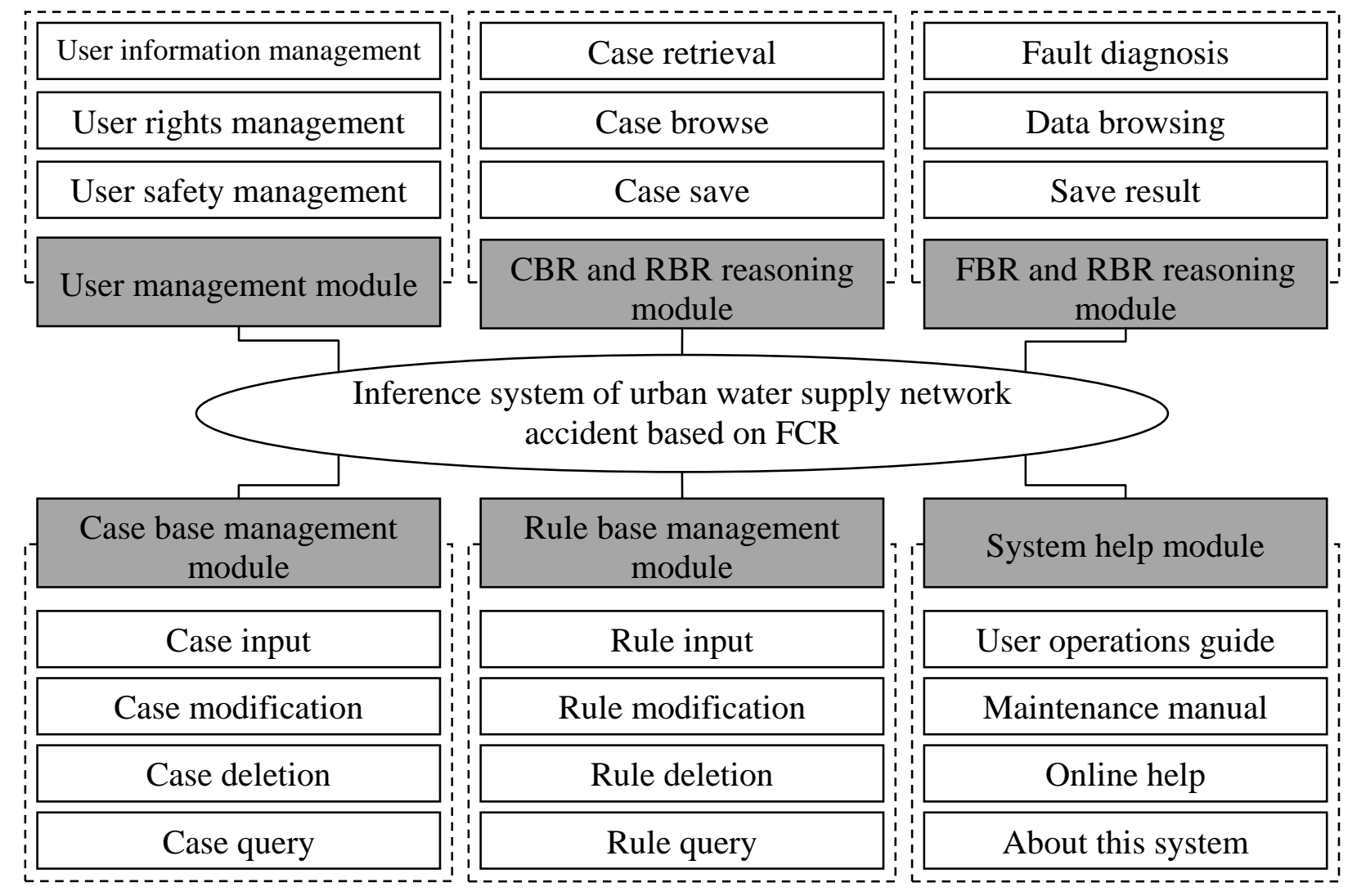

Fig. 4. System function structure

Both administrators and users need to enter account and password to login system, and then to the various modules, each module function is introduced as follows:

(1) Personnel management module. Mainly manage personnel information and authority of this system, including basic information management module and rights management module, the administrator can add, modify, and delete the basic information. 
(2) The CBR and RBR reasoning module. Users need to input the elements of water supply pipe data, case retrieval, and then submitted to the system after the retrieval, the user clicks on the "search results" button, the system will be according to the rules set by the fifth chapter will retrieve the results returned to the user for the browsing, the final user can save the target case and reasoning results to putted forward, the convenient use of reasoning in the future.

(3) The FBR and RBR reasoning module. When the users are not satisfied with the first reasoning, and reason the module. First and will the elements data input into the system, hand it over to the reasoning machine reasoning, then the inference results returned to the user in the form of text browsing, the final user can save the target case and reasoning results to putted forward, the convenient use of reasoning in the future. After the two types of reasoning, comparison results are obtained.

(4) Case management module. Case is rich, to a great extent, affect the execution efficiency, putted forward more rich more perfect, the reasoning efficiency is higher. So here with a particular user can manage the case, such as case input, modify, and delete operations, not only can reduce the redundant putted forward, at the same time also can make putted forward more and more rich, so as to improve the efficiency of reasoning.

(5) The rule base management module. Set up the aim of this module is in order to improve the level of rule base. When the fault information is becoming more and more libraries will be redundant or irrelevant rules, so you need to supervise and control the rule base for the users with specific privileges, such as query, input, modify, and delete rules of operation, has the function of maintenance and concise rule base.

(6) System help module. The module operates instructions for the system, and including how to use the system, how to carry out reasoning and rule base case bank and how to manage and so on.

\section{Conclusion}

Used in urban water supply pipeline accident reasoning fusion of CBR, RBR and FBR technology, both the advantages of CBR technology conforms to the human thinking process, CBR learning ability, and the FBR the advantages of easy maintenance and practical technology system, has the FBR simplicity, can well meet the accident reasoning system complexity characteristics of water supply pipe. Fusion reasoning raised the speed of the system of reasoning, reduce the error rate, and the reasoning can provide scientific guidance for water supply network accident reasoning, has a strong practical value.

\section{Acknowledgement}

This work is supported by 2014 general subject of colleges and universities scientific research of Liaoning province (L2014248): Emergency Disposal on Urban Water Supply Pipe Network Accident Based on FCR Computer Reasoning.

\section{References}

[1] J. W. Wang, "The technical research of emergent event's auto-process based on rule reasoning," Master's degree of Donghua University, 2009.

[2] Baidu Knows, "The introduction of fuzzy reasoning," https://zhidao.baidu.com/question/1497684157071800419.html, 2016-10-12.

[3] L. Li, H. M. Qi, F. X. Yang, "Research on traditional Chinese medicine diagnosis system based on case and fuzzy reasoning," Journal of Xinyang Normal University(Natural Science Edition), vol. 27, no. 4, pp. 585-588, 2014. 
[4] ZHAO Ying, ZHANG Jiao, REN Yong-chang, "Design on Emergency Disposal System for Urban Underground Water Supply Pipe Network based on FCR," Computer Knowledge and Technology, vol. 12, no. 26, pp. 253-255, 2016.

[5] ZHAO Ying, ZHANG Jiao, REN Yong-chang, "Research on Fusion Reasoning of 'Fuzzy + Case + Rule," Computer Knowledge and Technology, vol. 12, no. 25, pp. 230-232, 2016.

[6] Jeewon Seo, Moonsoo Koo, Kibum Kim, Jayong Koo, "A Study on the Probability of Failure Model Based on the Safety Factor for Risk Assessment in a Water Supply Network," Procedia Engineering, vol. 119, no. 5, pp. 206-215, 2015.

[7] Petra Perner, "Mining Sparse and Big Data by Case-based Reasoning," Procedia Computer Science, vol. 35, no. 2, pp. 19-20, 2014.

[8] N. Y. Xiang, K. L. Xin, Z. L. Yin, et al., "Emergency response and control strategy of sudden contamination accident in water supply network," Water Technology, vol. 7, no. 6, pp. 22-27, 2013. 